\title{
RATING THE DISCOUNT FOR A MOTOR INSURANCE EXCESS *
}

\author{
G. C. TAYLOR
}

The problem of rating the discount for an excess is considered under the condition where premium rates for excess-free policies are calculated according to a multiplicative (or equivalently, points rating) model.

Section 3 obtains some inequalities on the manner in which the discount should vary over the various risk-classes in the portfolio and compares the results with the current practical situation in Australia.

Section 4 derives a formula for rating the discount and gives some reasonable and practical approximations.

\section{DIFFICULTIES IN RATING THE DISCOUNT}

This paper considers the discount, or reduction in premium, which can be allowed in respect of an excess on a comprehensive motor insurance policy. In Australia such policies are limited almost entirely to property damage cover.

To establish terminology, let $E$ be the amount of the excess. Then, in the event of a claim involving damage of an amount $x$, the insurer is required to pay

$$
\max (0, x-E) \text {. }
$$

Most motor insurance portfolios include policies at various levels of excess, and so it may be thought that determining the discounts justified by these levels of excess would be a reasonably simple matter-merely a matter of examining the insurer's experience to discover the manner in which claims cost per vehicle varies with excess.

At this point one meets the perennial problem of motor insurance rating. It is that, according to the dictates of both experience and market practice, premiums for motor insurance are differentiated according to number of risk factors each of which can assume several values. This usually has the effect of producing so many subdivisions of the portfolio that the majority of them are statistically unreliable for rating purposes.

A further differentiation according to excess level obviously exacerbates this difficulty.

Furthermore, since many insurers overcome the difficulty discussed in the second last paragraph by using some mathematical technique, such as a multiplicative formula (discussed in more detail in Section 2), it is necessary to ensure in such cases that the manner in which a discount for excess is introduced is not such as to throw up anomalies relative to the rating formula.

* Presented at the $14^{\text {th }}$ ASTIN Colloquium, Taormina, October 1978. 


\section{A RATING MODEL (FOR A GIVEN EXCESS)}

For the present the question of excess will be ignored. Equivalently, it will be assumed that a unique excess applies to all risks insured in the portfolio.

It will be supposed, that there are $m$ risk factors, designated $R^{1}, R^{2}, \ldots, R^{m}$. It is supposed that $R^{i}$ can assume $n_{i}$ different values $R_{1}^{i}, \ldots, R_{n_{i}}^{i}$. Any particular risk is then described by the $m$-tuple of values assumed by the risk factors. For example, $\left(R_{j_{1}}^{1}, R_{j_{2}}^{2}, \ldots, R_{j_{m}}^{m}\right)$ will represent a risk for which the risk factor $R^{i}$ assumes the value $R_{j_{i}}^{i}, i=1,2, \ldots, m$.

Let $P\left(j_{1}, j_{2}, \ldots, j_{m}\right)$ denote the risk premium for this risk. In the sequel we shall make

\section{Assumption 1.}

The risk premium is defined by a multiplicative structure. More precisely, there exists a set of quantities $\left\{\pi_{j_{i}}^{i} i=1,2, \ldots, m ; j_{i}=1,2, \ldots, n_{i}\right\}$ such that the risk premium has the form:

$$
P\left(j_{1}, j_{2}, \ldots, j_{m}\right)=K \prod_{i=1}^{m} \pi_{j_{i}}^{i}
$$

As remarked in Section 1 this is a widely used rating device and often appears to fit the facts reasonably well. It is a device sometimes extended into a slightly different form which is more convenient administratively. The new form is obtained by noting that one may write

$$
\pi_{j_{i}}^{i}=(1+r)^{p_{j_{i}}^{i}}
$$

whence (2.1) becomes:

$$
P\left(j_{1}, \ldots, j_{m}\right)=K(1+r)^{\mathrm{e}},
$$

with

$$
\rho=\sum_{i=1}^{m} \rho_{j_{i}}
$$

It is usual to refer to $\rho_{j_{i}}$ as the points score associated with the value $R_{j_{i}}^{i}$ of the risk factor $R^{i}$. It is apparent from (2.4) that the risk premium is calculated by obtaining $p$, the total points score for the risk under consideration and raising the factor $(1+r)$ to this power.

\section{VARIATION OF DISCOUNT FOR EXCESS WITH RISK-CLASS}

Henceforth the $m$-tuple $\left(j_{1}, \ldots, j_{m}\right)$ describing the risk will be denoted by $\mathbf{j}$ in vector notation.

Let $\lambda(\mathbf{j})$ be the claims frequency associated with risk $\mathbf{j}$ and let $F(. \mid \mathbf{j})$ be the corresponding d.f. of claim sizes. Then

$$
P(\mathbf{j})=\lambda(\mathbf{j}) \mu(\mathbf{j}),
$$


where

$$
\mu(\mathbf{j})=\int_{0}^{\infty} x d F(x \mid \mathbf{j})
$$

which is the mean claim size associated with $\mathfrak{j}$.

We now wish to introduce an excess into the rating system. Let $P_{E}(\mathbf{j})$ be the risk premium required under the action of an excess $E$,

$$
P_{E}(\mathbf{j})=\lambda(\mathbf{j}) \mu_{E}(\mathbf{j}),
$$

where

$$
\begin{aligned}
\mu_{E}(\mathbf{j}) & =\int_{E}^{\infty}(x-E) d F(x \mid \mathbf{j}) \\
& =\int_{E}^{\infty} H(x \mid \mathbf{j}) d x
\end{aligned}
$$

where $H$ is the complementary d.f.

$$
H(x \mid \mathbf{j})=1-F(x \mid \mathbf{j})
$$

The difference between the premium rate applicable in the absence of an excess and that applicable in the presence of an excess of $E$ is called the discount for excess $E$. If it is denoted by $D_{E}(\mathbf{j})$, then

$$
\begin{aligned}
D_{E}(\mathbf{j}) & =P_{0}(\mathbf{j})-P_{E}(\mathbf{j}) \\
& =\lambda(\mathbf{j})\left[\mu_{0}(\mathbf{j})-\mu_{E}(\mathbf{j})\right] \\
& =\lambda(\mathbf{j}) \int_{0}^{E} H(x \mid \mathbf{j}) d x .
\end{aligned}
$$

The first qualitative question in which one might be interested is how $D_{E}$ responds to changes in $\mathbf{j}$ (for given $E$ ). Clearly, without any assumptions about the way in which $\lambda$ and $F$ vary with $\mathbf{j}$, practically any type of response of $D_{E}$ is possible. In order to cut down on the range of possibilities and gain some insight into the problem, we make a distributional assumption.

\section{Assumption 2.}

The same distribution of claim sizes occurs in risk-classes $\mathbf{j}$ and $\mathbf{k}$ except that the claim sizes differ by a scalar multiple. More precisely,

$$
F(x \mid \mathbf{j})=F(\alpha(\mathbf{j}) x),
$$

for some d.f. $F$ which is independent of $\mathbf{j}$.

It should perhaps be pointed out that the meaning of the scaling constant $\alpha(\mathbf{j})$ is that $\alpha(\mathbf{j})>\alpha(\mathbf{k})$ means that the $\mathbf{j}$ amount is stochastically smaller than the $\mathbf{k}$ amount. 
Note that

[by (3.2)]

$$
\begin{aligned}
\mu_{0}(\mathbf{j}) & =\int_{0}^{\infty} H(\alpha \cdot(\mathbf{j}) x) d x \\
& =\int_{0}^{\infty} H(y) d y / \alpha(\mathbf{j}) \\
& =\mu_{0} / \alpha(\mathbf{j}),
\end{aligned}
$$

where $\mu_{0}$ is the mean associated with the d.f. $F$. Thus

$$
P_{0}(\mathbf{j})=\mu_{0} \lambda(\mathbf{j}) / \alpha(\mathbf{j}) .
$$

Now

[by (3.3)]

$$
\begin{aligned}
D_{E}(\mathbf{j}) & =\lambda(\mathbf{j}) \int_{0}^{E} H(x \mid \mathbf{j}) d x \\
& =\lambda(\mathbf{j}) \int_{0}^{E} H(\alpha(\mathbf{j}) x) d x \\
& =[\lambda(\mathbf{j}) / \alpha(\mathbf{j})] \int_{0}^{E x(\mathbf{j})} H(y) d y .
\end{aligned}
$$

Therefore, by substitution of (3.5) into (3.6):

$$
D_{E}(\mathbf{j})=\mu_{0}^{-1} P_{0}(\mathbf{j}) \int_{0}^{B \alpha(\mathbf{j})} H(y) d y .
$$

We now wish to compare the size of the discount for different values of $\mathbf{j}$. From (3.1), if $\alpha(\mathbf{j})>\alpha(\mathbf{k})$, then

$$
\begin{aligned}
D_{E}(\mathbf{j}) & =\lambda(\mathbf{j}) \int_{0}^{E} H(\alpha(\mathbf{j}) x) d x \\
& =\lambda(\mathbf{j})[\alpha(\mathbf{k}) / \alpha(\mathbf{j})] \int_{0}^{E \alpha(\mathbf{j}) / \alpha(\mathbf{k})} H(\alpha(\mathbf{k}) y) d y \\
& \geqslant \lambda(\mathbf{j})[\alpha(\mathbf{k}) / \alpha(\mathbf{j})] \int_{0}^{E} H(\alpha(\mathbf{k}) y) d y \\
& =[\lambda(\mathbf{j}) / \alpha(\mathbf{j})][\alpha(\mathbf{k}) / \lambda(\mathbf{k})] D_{E}(\mathbf{k}),
\end{aligned}
$$

by (3.6).

Using (3.5) in (3.8):

$$
\frac{D_{E}(\mathbf{j})}{D_{E}(\mathbf{k})} \geqslant \frac{P_{0}(\mathbf{j})}{P_{0}(\mathbf{k})} .
$$


It may be noted that equality holds here if and only if there is zero probability of obtaining in risk-class $\mathbf{k}$ a claim size between $E$ and $E \alpha(\mathbf{j}) / \alpha(\mathbf{k})$. Thus, for practical cases we may take

$$
\frac{D_{E}(\mathbf{j})}{D_{E}(\mathbf{k})}>\frac{P_{0}(\mathbf{j})}{P_{0}(\mathbf{k})}
$$

Returning to (3.6):

$$
\begin{aligned}
D_{E}(\mathbf{j}) & =[\lambda(\mathbf{j}) / \alpha(\mathbf{j})] \int_{0}^{E \alpha(\mathbf{j})} H(y) d y \\
& =[\lambda(\mathbf{j}) / \alpha(\mathbf{j})][\alpha(\mathbf{j}) / \alpha(\mathbf{k})] \int_{0}^{E \alpha(\mathbf{k})} H(x \alpha(\mathbf{j}) / \alpha(\mathbf{k})) d x \\
& \leqslant[\lambda(\mathbf{j}) / \alpha(\mathbf{k})] \int_{0}^{E \alpha(\mathbf{k})} H(x) d x \\
& =[\lambda(\mathbf{j}) / \lambda(\mathbf{k})] D_{E}(\mathbf{k}) .
\end{aligned}
$$

Again we note that equality holds only if in both risk-classes $\mathbf{j}$ and $\mathbf{k}$ there is zero probability of a claim below the excess. Thus, combining (3.10) and (3.11) for practical cases:

$$
\frac{P_{0}(\mathbf{j})}{P_{0}(\mathbf{k})}<\frac{D_{E}(\mathbf{j})}{D_{E}(\mathbf{k})}<\frac{\lambda(\mathbf{j})}{\lambda(\mathbf{k})} \text { for } \alpha(\mathbf{j})>\alpha(\mathbf{k})
$$

Now it is common in motor insurance to find that $\lambda(\mathbf{j})$ and $\mu_{0}(\mathbf{j})$ vary in sympathy as $\mathbf{j}$ varies, i.e.

$\lambda(\mathbf{j})<\lambda(\mathbf{k})$ if and only if $\mu_{0}(\mathbf{j})<\mu_{0}(\mathbf{k})$. It then follows from (3.4) and (3.5) that

$$
P_{0}(\mathbf{j})<P_{0}(\mathbf{k}) \text { if and only if } \alpha(\mathbf{j})>\alpha(\mathbf{k}) .
$$

In this case (3.12) can be replaced by:

$$
\frac{P_{0}(\mathbf{j})}{P_{0}(\mathbf{k})}<\frac{D_{E}(\mathbf{j})}{D_{E}(\mathbf{k})}<\frac{\lambda(\mathbf{j})}{\lambda(\mathbf{k})}<1,
$$

if and only if $P_{0}(\mathbf{j})<P_{0}(\mathbf{k})$.

What this means in graphic terms is illustrated in the following diagram.

In this diagram, risk-classes are arranged along the horizontal axis in such a way that the premium rates, plotted against the premium rate for a standard risk-class, form a linear graph. The shaded area shows the area of the graph in which the discount for excess $E$, also expressed as a percentage of the discount for the standard risk-class, can lie.

The result embodied in (3.13) and the diagram is an interesting one because, in Australia at least, there is currently a strong tendency to allow a constant or near-constant discount for all risk-classes. This seems difficult to justify on the facts. 


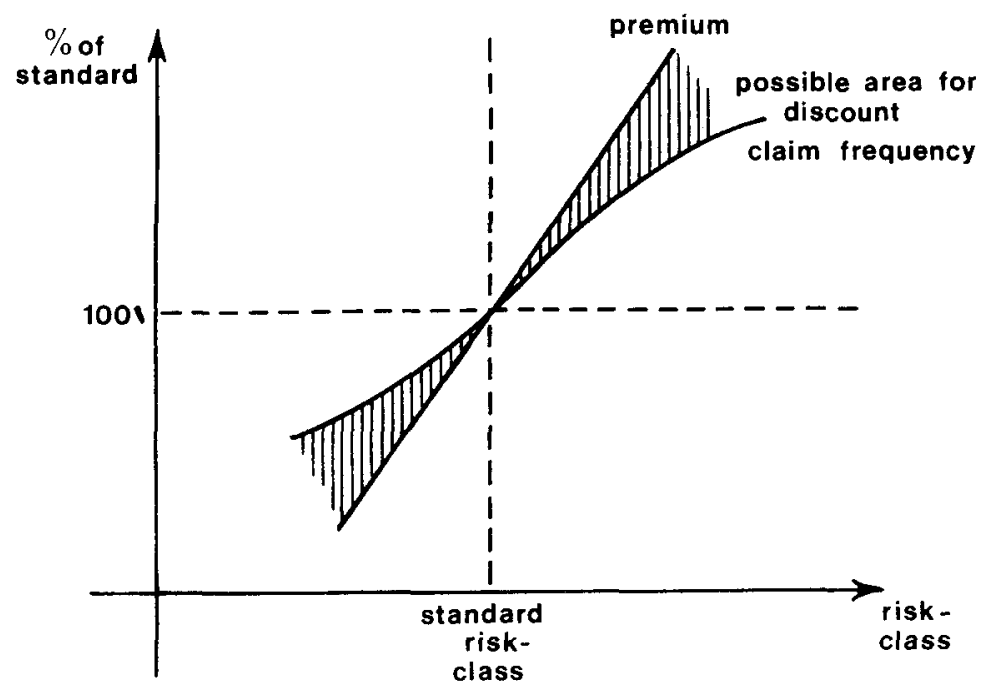

4. CALCULATING THE DiscoUnt

Equation (3.3) gives an expression for the discount for excess $E$ under quite general conditions. Clearly, it is difficult to proceed in the absence of a knowledge of all the d.f.'s $F(. \mid \mathbf{j})$ unless some other assumptions are made. In this section we retain Assumption 1 from Section 2. We also make the assumption that, over all the risk-classes, claim frequency and average claim size vary in sympathy in a specific way, i.e.

\section{Assumption 3 .}

$\mu_{0}(\mathbf{j}) / \mu_{0}(\mathbf{k})=[\lambda(\mathbf{j}) / \lambda(\mathbf{k})]^{\beta}$, for some constant $\beta$.

By (3.1),

$$
\frac{P_{0}(\mathbf{j})}{P_{0}(\mathbf{k})}=\left[\frac{\lambda(\mathbf{j})}{\lambda(\mathbf{k})} \frac{\mu_{0}(\mathbf{j})}{\mu_{0}(\mathbf{k})}\right]=\left[\frac{\lambda(\mathbf{j})}{\lambda(\mathbf{k})}\right]^{1+\beta}
$$

by Assumption 3. Then, by (2.3) and (4.1),

$$
\begin{aligned}
\frac{\lambda(\mathbf{j})}{\lambda(\mathbf{k})} & =\left[\frac{(1+\gamma)^{\rho(\mathbf{j})}}{(1+r)^{\rho(\mathbf{k})}}\right]^{1 /(1+\beta)} \\
& =(1+\gamma)^{(\rho(\mathbf{j})-\rho(\mathbf{k})) /(1+\beta)}
\end{aligned}
$$

where

$$
\rho(\mathbf{j})=\sum_{i \sim 1}^{n} \rho_{j_{i}}^{i}
$$

which is an alternative form of (2.4). 
Now by $(4 \cdot 2)$ and $(4 \cdot 3)$

$$
\frac{\lambda(\mathbf{j})}{\lambda(\mathbf{k})}=\prod_{i=1}^{m}(1+\gamma),{ }^{\left(\rho_{j_{i}}^{i}-\rho_{k_{i}}^{i}\right) /(1+\beta)}
$$

which means that claim frequency has a multiplicative structure. It follows immediately from (4.4) and Assumption 2 that average claim size has a multiplicative structure also.

We now revert to $(3.6)$ :

$$
\begin{aligned}
D_{E}(\mathbf{j}) & =[\lambda(\mathbf{j}) / \alpha(\mathbf{j})] \int_{0}^{E \alpha(\mathbf{j})} H(y) d y \\
& =\left[\mu_{0} \lambda(\mathbf{k}) / \alpha(\mathbf{k})\right] \frac{\lambda(\mathbf{j})}{\lambda(\mathbf{k})} \frac{\alpha(\mathbf{k})}{\mu_{0}} \int_{0}^{E \alpha(\mathbf{j})} H(y) d y / \alpha(\mathbf{j}) \\
& =\frac{\lambda(\mathbf{j})}{\lambda(\mathbf{k})} P_{0}(\mathbf{k}) \int_{0}^{B \alpha(\mathbf{j})} H(y) d y / \mu_{0}(\mathbf{k}) \alpha(\mathbf{j}),
\end{aligned}
$$

by (3.4) and (3.5). Thus, by (4.2),

$$
D_{E}(\mathbf{j})=\gamma(\mathbf{j}) P_{0}(\mathbf{k})\left[\frac{(1+r)^{\rho(\mathbf{j})}}{(1+\gamma)^{\rho(\mathbf{k})}}\right]^{1 /(1+\beta)}
$$

where

$$
\gamma(\mathbf{j})=\frac{E}{\mu_{0}(\mathbf{k})} \int_{0}^{E \alpha(\mathbf{j})} H(y) d y / E \alpha(\mathbf{j}) .
$$

Now, as is apparent from (2.1)-2.4), it is possible to choose $K=P(\mathbf{k})$, say, so that $\rho(\mathbf{k})=0$, i.e. $\mathbf{k}$ is a risk-class with zero points score.

Then (4.5) becomes

$$
D_{E}(\mathbf{j})=\gamma(\mathbf{j}) P_{0}(\mathbf{k})(1+\gamma)^{\rho(\mathbf{j}) /(1+\beta)},
$$

where $\gamma$ is defined by (4.6).

\subsection{First approximation to the discount.}

Normally $E \alpha(\mathbf{j})$ will be fairly small in relation to $\mu_{0}$ (perhaps of the order of $10 \%$ ) and $H(y)$ will vary fairly smoothly for small values of $y$. Therefore, a first approximation to $\gamma(\mathbf{j})$ is

$$
\gamma(\mathbf{j})=\left[E / \mu_{0}(\mathbf{k})\right] H\left(\frac{1}{2} E \alpha(\mathbf{k})\right),
$$

or even

$$
\gamma(\mathbf{j})=E / \mu_{0}(\mathbf{k})
$$


The second of these has the advantage of being simple. It has the disadvantage of overestimating the discount. Equation (4.1.1) is preferable, and an unsophisticated examination of claim sizes should be sufficient to form an idea of the size of $H\left(\frac{1}{2} E \alpha(\mathbf{k})\right)$.

\subsection{Second approximation to the discount}

The disadvantage of approximations (4.1.1) and (4.1.2) is that both disregard the dependence of $(\mathbf{j})$ on $\mathbf{j}$. This can be taken into account by means of the approximation :

$$
\begin{aligned}
\gamma(\mathbf{j}) & =\left[E / \mu_{0}(\mathbf{k})\right]\left[1+E \alpha(\mathbf{j}) H^{\prime}\left(\frac{1}{2} E \alpha(\mathbf{j})\right)\right] \\
& =\left[E / \mu_{0}(\mathbf{k})\right]\left[1+E H^{\prime}\left(\frac{1}{2} E \alpha(\mathbf{j})\right) \mu_{0} / \mu_{0}(\mathbf{j})\right] \\
(4.2 .1) & =\left[E / \mu_{0}(\mathbf{k})\right]\left\{1+\mu_{0} H^{\prime}\left(\frac{1}{2} E \alpha(\mathbf{j})\right)\left[E / \mu_{0}(\mathbf{k})\right](1+\gamma)^{-\rho(\mathbf{j}) \beta_{3}(1+\beta)}\right\}
\end{aligned}
$$

by Assumption 3 and (4.2).

\subsection{Rule for calculation of discount}

The verbal statement of calculation of the discount for excess $E$ in risk-class $\mathbf{j}$ is as follows:

1. Calculate the points score for risk-class $\mathbf{j}$.

2. Divide this points score by the constant $(1+\beta)$.

3. Raise $(1+r)$ to that power.

4. Multiply the result by $\gamma$ times the excess-free premium rate for riskclasses with zero points score. Note that $\gamma$ is defined in (4.1.1), (4.1.2) or (4.2.1) in terms of the mean claim size in risk-classes with zero points score.

Note that our expressions for the discount, in the form of (4.5), satisfy the inequality (3.13).

\section{ESTIMATION OF THE PARAMETER}

By Assumption 3,

$$
\log \mu_{0}(\mathbf{j})=\beta \log \lambda(\mathbf{j})+\text { const., }
$$

which suggests that, as a simple measure, $\beta$ might be estimated as the regression coefficient of $\log \mu_{0}(\mathbf{j})$ on $\log \lambda(\mathbf{j})$. More efficient estimators could be found, e.g. maximum likelihood, which would be asymptotically efficient.

E. S. Knight and Co., Sydney, Australia. 